\title{
Statistical analysis of spinal cord injury severity detection on high dimensional MRI data
}

\author{
Sk Hasane Ahammad', V Rajesh ${ }^{2}$, K Saikumar ${ }^{3}$, Sridevi Jalakam ${ }^{4}$, G.N.S. Kumar ${ }^{5}$ \\ $1,2,3,5$ Department of Electronics and Engineering, Koneru Lakshmaiah Education Foundation, India \\ ${ }^{4} \mathrm{NRI}$ Insitute of Technology, India
}

\begin{abstract}
Article Info
Article history:

Received Mar 22, 2019

Revised Apr 3, 2019

Accepted Apr 12, 2019

Keywords:

Filtered segmentation

Multilevelsegmentation (MLS)

SCI (Spinal Cord Injury)

ABSTRACT

Staggered Segmenting on the programmed spinal rope form is a vital advance for evaluating spinal line decay in different infections. Outlining dark issue (GM) and white issue (WM) is additionally helpful for measuring GM decay or for extricating multiparametric MRI measurements into WMs tracts. Spinal line division in clinical research isn't as created as cerebrum division, anyway with the considerable change of MR groupings adjusted to spinal line MR examinations, the field of spinal rope MR division has progressed extraordinarily inside the most recent decade. Division strategies with variable exactness and level of multifaceted nature have been produced. In this paper, we talked about a portion of the current strategies for line and WM/GM division, including power based, surface-based, and picture based and staggered based techniques. We likewise give suggestions to approving spinal rope division systems, as it is essential to comprehend the inborn qualities of the strategies and to assess their execution and constraints. In conclusion, we represent a few applications in the solid and neurotic spinal string. In this task, an Automatic Spinal Cord Injury (SCI) is identified utilizing a staggered division technique.
\end{abstract}

Copyright @ 2019 Institute of Advanced Engineering and Science. All rights reserved.

\section{Corresponding Author:}

Sk Hasane Ahammad,

Department of Electronics and Communication Engineering,

Koneru Lakshmaiah Education Fondation,

Guntur-522502, India.

Email: ahammad@kluniversity.in

\section{INTRODUCTION}

Exact arranging of radiation treatment involves the meaning of treatment volumes and a reasonable delimitation of ordinary tissue of which pointless presentation ought to be counteracted this is possible with segmentation [1]. The spinal rope is a radiosensitive organ, which ought to be unequivocally distinguished because an Overexposure to radiation may prompt undesired inconveniences for the patient, for example, neuronal disfunction or loss of motion. In this work, an information-based way to deal with distinguishing the spinal rope in figured tomography pictures of the thorax is exhibited. The approach depends on an information base which comprises of an assumed anatomical structure delineate and an assignment arranged design called the automatic SCI [2]. The ASM (automatic spinal card injury finding mechanism) contains a casing like learning portrayal of the full-scale life structures in the human thorax. The arrangement solver oversees deciding the position, introduction and size of the structures important to radiation treatment. The arrangement solver depends on various picture preparing administrators. Some are alleged nuclear (e.g., thresholding and snakes) though others are composite. The entire framework has been actualized on a standard PC. Examinations performed on the picture material from 3 patients demonstrate that the approach brings about a solid acknowledgment of the spinal rope (92\% precision) and the spinal trench (85\% exactness) [3-4]. The lamina is riskier to find accurately (precision 72\%). The situation of the external thorax is constantly decided effectively. For a couple of therapeutic pictures, it isn't possible to set division 
process parameters, for instance, edge regard, so every one of the objects of interest are removed from the establishment or each other without over-dividing the data. Over-division is the system by which the articles being administered by the establishment are themselves divided or broke into subcomponents. While over-division constructs the chances that cutoff points of hugeness have been evacuated, it does accordingly at the cost of making various insignificant breaking points. For this circumstance, pre-separating methodology, as inspected in past areas, should be used as a piece of an undertaking to wipe out the disturbance, improve between question definition, or smooth picture surfaces, all of which may cause division inconveniences. In case these systems are not sufficient, over-division can be used as a starter getting ready advance, trailed by social affair shapes that undertaking to reassemble the articles into single picture events (one inquiry) [5]. Each part got from the photo is insinuated as a challenge, and the properties of the bits are allocated as attributes or features.

After a photograph is apportioned, its flanking regions can be converted to shape a less isolated division considering their closeness. Similarity estimations can be depicted as a fragment of force, shading, check, surface, change, and shared edge degree, among others. What's more, for any part, indisputable strategies can be prompted to quantify likeness. For example, the refinement of control between circumscribing articles can be evaluated by two frameworks. In one strategy, the common of all the pixel controls in a single test can veer from the regular force of the noteworthy number of pixels in a neighbouring article. This procedure is named differentiation. On the other hand, neighbourhood complexity can be taken care of by looking at ordinary power made up of basically the pixels that are adjoining the fringe between two articles. Either or the best of the two can be utilized. For another decision, the measure of the articles being emerged could be utilized to choose which isolate framework is utilized [6].

The general differentiation can be enrolled when little request are being considered, and neighborhood partition can be utilized for sweeping articles. A second technique is select the detachment estimation framework considering the refinement of the different powers. Where tremendous power differentiate exists, near to detachment may be all the all the more fitting, while generally capriciousness could be utilized for objects that have low power change. It is the objective of a closeness work (f) to assess the resemblance of two contiguous difficulties in a photograph. Right when all is said in done, the more highlights this cutoff considers, the more satisfactory the outcomes it produces. A proportionality score can along these lines be settled as the farthest point $(\mathrm{F})$ of different likenesses sub-limits (fn): Closeness Score $=F(f 1 w 1, f 2 w 2, f 3 w 3, \ldots)$ where $F$ is the blend work, which can be an improvement, duplication, most crazy, smallest, and so forth, and wn is weight used to set the criticalness of every comparability highlight. The utilization of weights makes the joining framework versatile. That is, the joining of things can be controlled by the set of weights by the client at runtime or can be picked subsequently by the vision framework in light of other operational parameters, for example, the kind of information being dealt with, basic conditions, clamour levels, et cetera. This framework is a decent case of how a dream structure can be made astute [7].

The systems under trade are strikingly stimulated by the accessibility of instruments for emblematic delineation and dealing with. That is, the vision organizer should approach a strategy of programming devices that give portrayals and to a database-association framework for pictures and their pixel-based delineation, and for the geometric shapes and characteristics of these articles, which are from time to time hinted as highlights. Test adjacencies can be anchored in such a significant database as affiliations, and similarity scores can be anchored as characteristics of the affiliations [8]. Sensibly, the affiliations and the attributes can be imagined as sensible like structures, where each inside in the truth is a test in the photograph, and the relationship in the down to earth show the closeness of articles in the photograph.

a. Multi Level Segmentation Technique

This region demonstrates the most apropos works in the field of examined picture division, underlining the ones most related to remedial applications. They are designated strange state and low-level. Related works following practically identical philosophies are indicated too. Moreover, a portrayal of estimations for division quality is displayed. Finally, the proposed approach is displayed. Around there, a delegate set of existing division estimations is broke down and a brief portrayal of some of them is given. The inspiration driving this section is to give the per client a blueprint of the bleeding edge in restorative picture division, especially the ones related to remote distinguishing. The written work contains a couple of extraordinary outlines about picture division philosophies proposed in the latest decades.

The principle requested division approaches, made dull scale pictures, into three classes: gathering, edge acknowledgment and territory extraction. The second assessed additionally astounding division techniques introduced feathery/non-fleecy instruments, Markov Random Fields (MRFs) probabilistic models, shading information and furthermore neural frameworks each one of them were still in their starting circumstances of headway. Among the essential that exclusively gave an all-around chart of computations centered at administering shading pictures. Here presented an expansive outline of picture division 
examination occurred in this work, they amass shading picture division figuringsin light of their particular foundation (low level logical classification). Along these lines, picture division techniques can be thoroughly requested as shown in Figure 1 considering: 1. the photo compose, 2. the level of human coordinated effort, 3. how the photo is addressed for getting ready, 4. the number and sort of qualities used and 5. The fundamental standard of movement. The last one isolates division computations as being either spatially outwardly debilitated or spatially guided, dependent upon paying little heed to whether spatial information is abused. Further, a low-level logical characterization was proposed as shown in Figure 1, which especially bundles division procedures in light of their particular sections.

The assurance of a division computation when in doubt relies upon the envisioned application. For instance, MRI SCAN Thresholding based division is a not too bad decision for diminish scale pictures, for instance, remedial pictures (tomography, $\mathrm{X}$ bars, et cetera.). In any case, it isn't so predominant in remote SCI zones especially in finding harm applications as a result of the abnormal state of variability of extraordinary features. As the goal of this examination are restorative applications, this work bases on division approaches significant to this field.One of the continuous examinations made to arrange spinal picture division was coordinated by Image Processing based Segmentation Techniques for Spinal Cord in MRI [9]. This work gives learning into the essential mechanical parts of picture division of stunned remote identifying pictures. In light of them, the typical division approaches SCI are included in Figure 1 (Blue Square shapes). In the going with areas, related endeavors to the picked division estimations will be investigated. Gathering based division counts are spatially outwardly debilitated frameworks wherein the photo data is viewed as a point cloud on a one-dimensional (1-D) dim scale center or in a multidimensional shading space dependent upon the photo makes.

The best favored outlook of collection approaches is their natural ease and straightforwardness of execution. Regardless, the guideline issue is to describe an appropriate number of groups, especially when the dimensionality of the part space is high. A couple of instances of packing-based procedures are MeanShift division, Self-Organizing Map (SOM) Neural Networks (Fuzzy-based) among others. SOMs have not gotten much thought in remote recognizing. In any case, they have wide applications in therapeutic imagery, with various types of Artificial Neural Networks (ANN, for instance, Multilayer Perceptron (MLP) and Hopfield Network. Zone based systems frequently use traditions including growing, part and mixing, solely or in sensible blends [10]. Region creating deduces a method that starts from a single pixel or little predefined checked game plan of pixels called "seed" and, in perspective of a particular homogeneity standard, iteratively adds up to pixels around it. Region part is a framework that is begun with an inhomogeneous division of a photo, which is needlessly part until the point when the moment that pieces satisfying a homogeneity demonstrate are obtained. Region uniting is a system in which sub-areas conceivably part of a broad identifiable region are interwoven to yield a reduced course of action of segments that are spatially critical with respect to the information picture content. Instances of zone-based philosophies associated with remote distinguishing have been made here.

b. To minimize the over segmentation regions on the complex SCI images using MLS

MLS is a typical strategy for picture division. Be that as it may, its utilization for medicinal picture division has been constrained especially due to over-division. Considering the attributes of a medicinal picture, particularly the form extraction from the MRI (attractive reverberation imaging) SPINAL picture, this work proposes an enhanced technique keeping in mind the end goal to defeat the downsides. Right off the bat, multi-scale exchanging consecutive sifting by reproduction is acquainted with taking out the commotion and streamline the information pictures, and the loss of limit data can be maintained a strategic distance from [11] segmentation. Also, two techniques for h-minima and minimal burden are forced on the inclination picture to stamp the minimal areas, so the entirety of its nearby minima are smothered. At long last, the MLS is connected to the stamped slope pictures to get the shape of SPINAL. Exploratory outcomes demonstrate that the enhanced strategy can be connected to form extraction of a spinal-cord picture with the great outcome, and the mean lessening of nearby minima in the over-portioned picture of districts is the great contrast with the watershed change in view of check extraction old technique.

The proposed technique has been executed and tried on the MRI cerebrum picture information from diagnosis centres, healing centre with just about 160 cuts for everyone in addition to 3 people. The checking mechanical assembly is Philips MR scanner and cut thickness is $1.5 \mathrm{~mm}$. To show the proficiency of the proposed strategy, a few tests have been completed on the pictures with various attributes. Right off the bat, the examination is about the shape extraction of MRI spinal card picture. These new programmed SCI techniques can be connected to contemplate demyelination and degeneration in different spinal string sicknesses, for example, MS or cervical myelopathy. 


\section{ALGORITHM}

Here image type means whether it is a medical or any type and monochrome means that have same properties. Colour represents that weather is GM or WM. Human Interaction is the technique just see and get an idea what change cloud happen. Image representation contains single scale and multi scale modules here divided the image into segments based on pixels and comparison methods. Coming to image attributes consists of single and multi attribute. The principle of operation consists of spatially blind and spatially guided modules they are the main blocks of multi level segmentation (MLS) [12] these can be explained in Figure 1. Figure 2 explains that different types of intensity type andSurface based, and image based technical multi-level extraction are applied on the medical image and got good results rather than over segmentation effects. Results shown below.

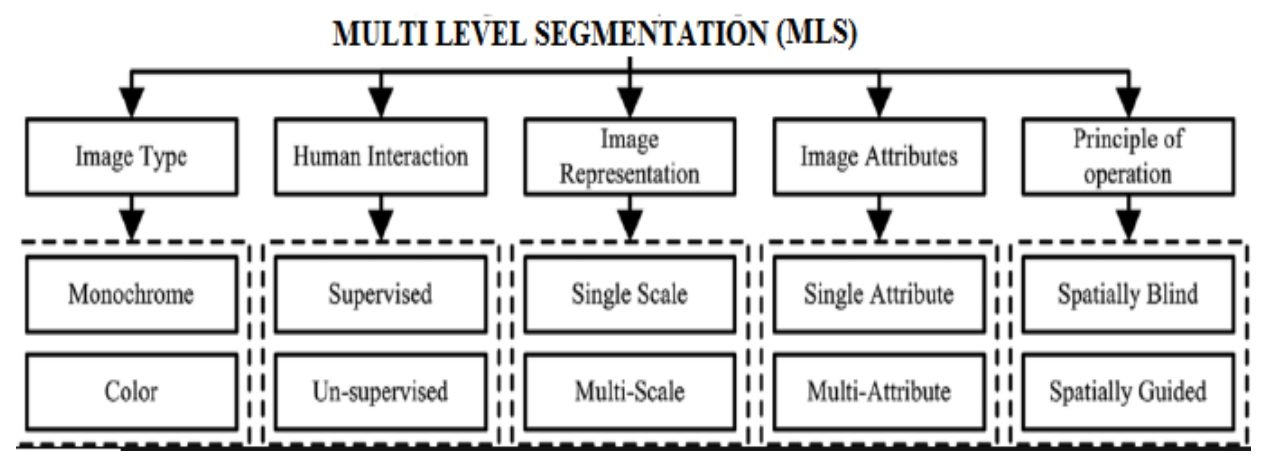

Figure 1. Multi-level segmentation block diagram

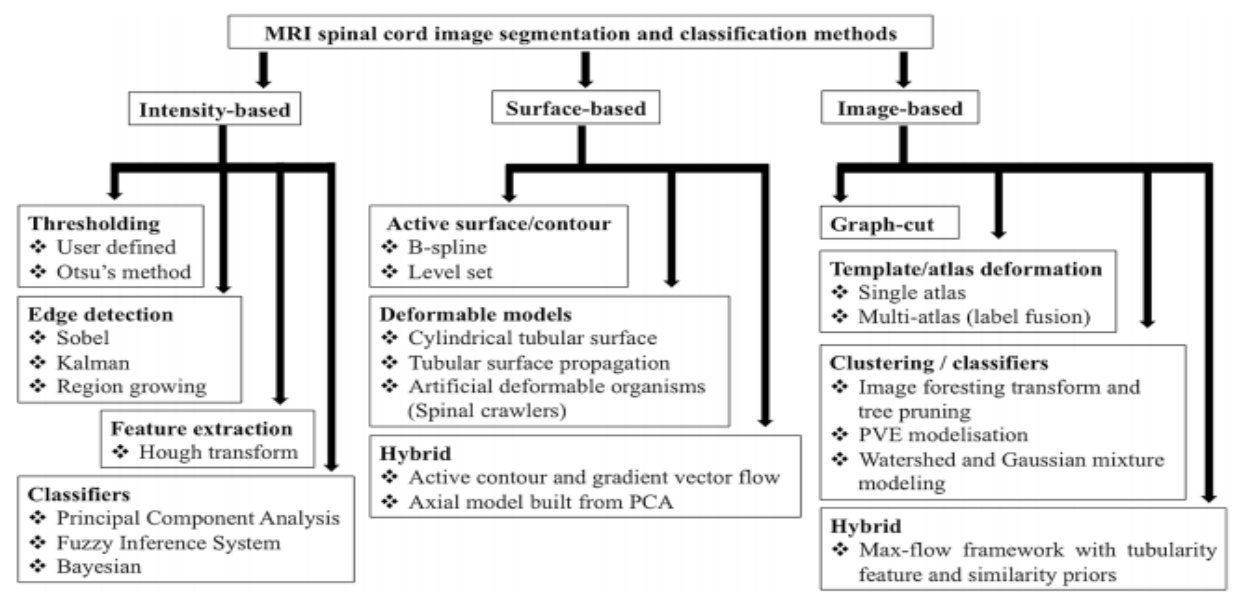

Figure 2. Multi level functions

\section{EXPERIMENTAL RESULTS}

There are two spinal nerves that are particularly vulnerable to injury - the radial and sciatic these also found in this MLS method. Crutches that are improperly adjusted can sometimes cause crutch paralysis by compressing the radial nerve, which passes through the underarm, against the upper arm bone.

Figure 3 explains that spinal cord automatic SCI detection image here we avoid the over segmentation problem using MLS algorithm. Figure 4 is the patient-1 information. Here we observe the image clearly every bone of SCI is visible, so this method is good comparative previous methods. So, this is the best proposed algorithm comparative to low level extraction. All sub level extractions are applied to selected image and ie patient $1 \& 2$. Here Figure 5 and Figure 6 shows data of echo train length and absorption rate. Using this data we conclude that selected techniques is best compared all other methods. 

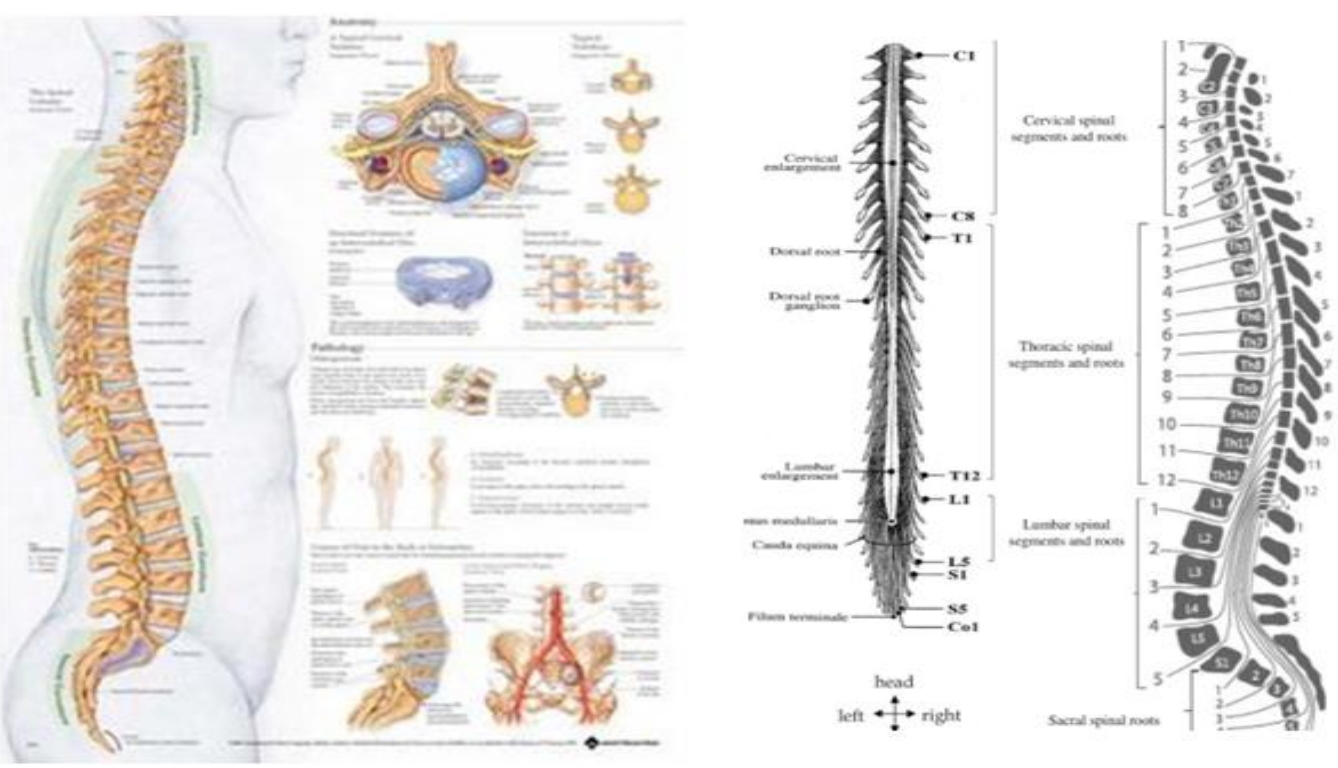

Figure 3. Human bodies focus on spinal card

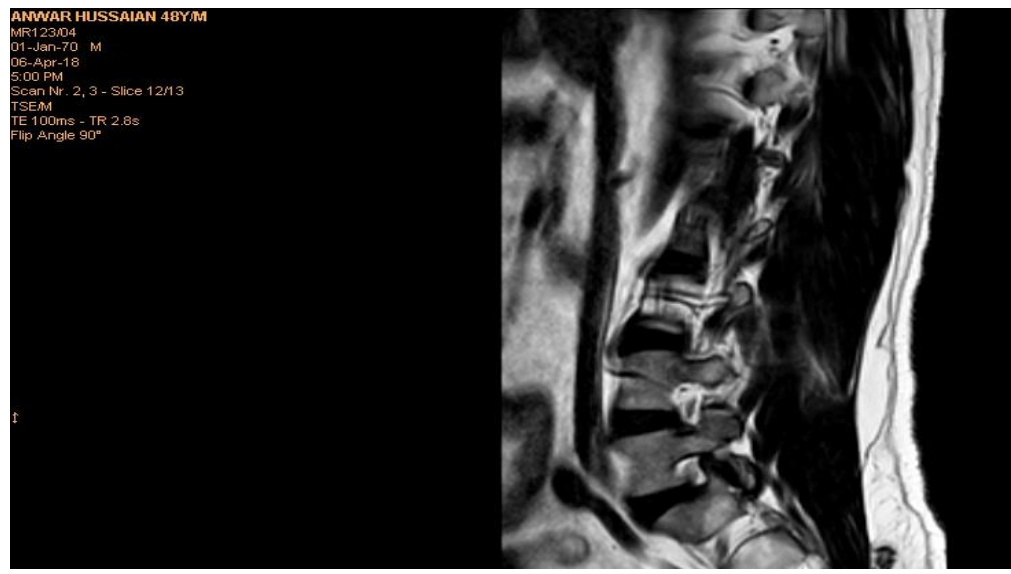

Figure 4. Patient -1 spinal cord

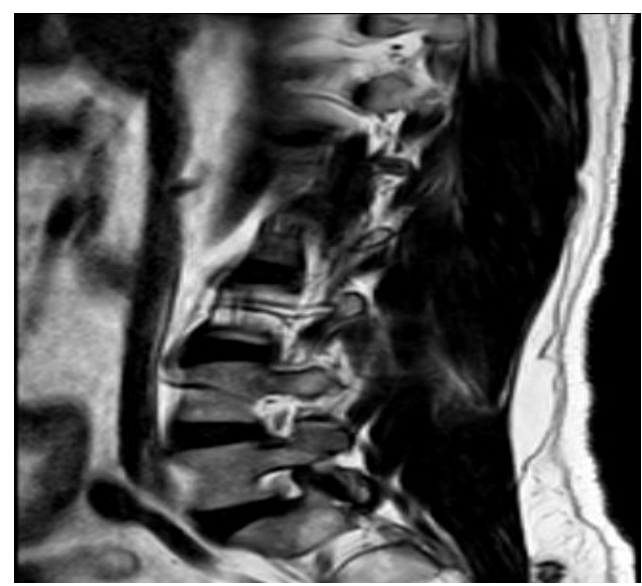

Figure 5. Detail images of Figure 3 sub 


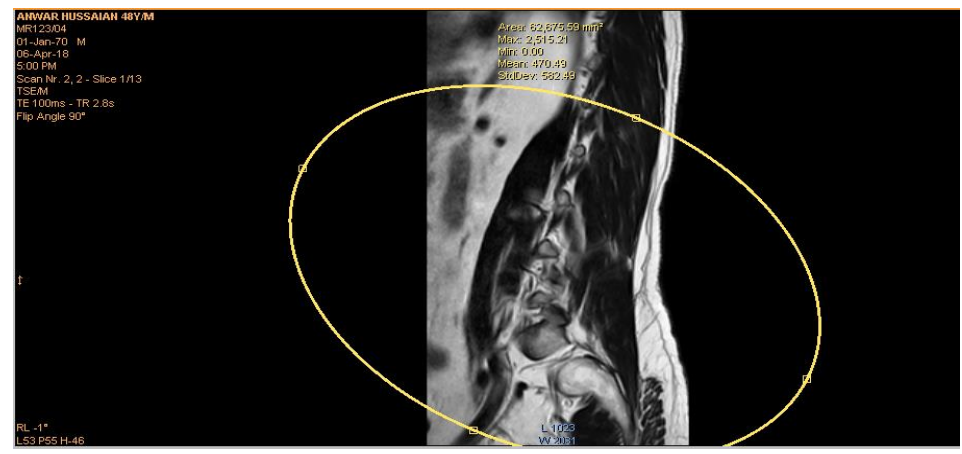

Figure 6. Spinal cords of second patient

Figure 7 describes the multi-level segmentation dataset of the training spinal cord image data. In the figure, segmentation method is applied on different types of noises in order to remove the noise before tracking the actutal segmentated regions. Figure 8, describes the multi-level segmentation dataset of the training spinal cord image data. In the figure, segmentation training data and its statistical properties of each attribute is given to validate the spinal cord regions.

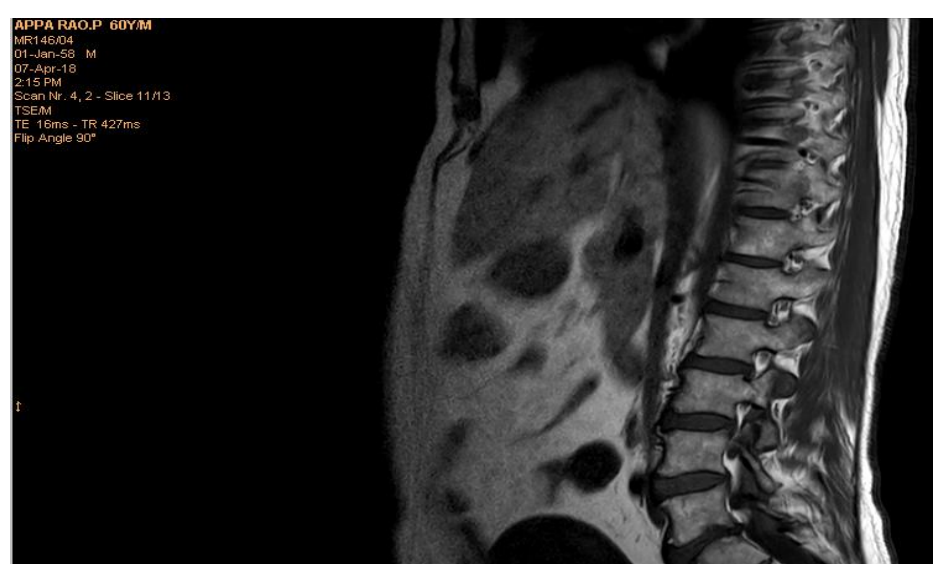

Figure 7. Clear multi level segmentation process of patient-spinal

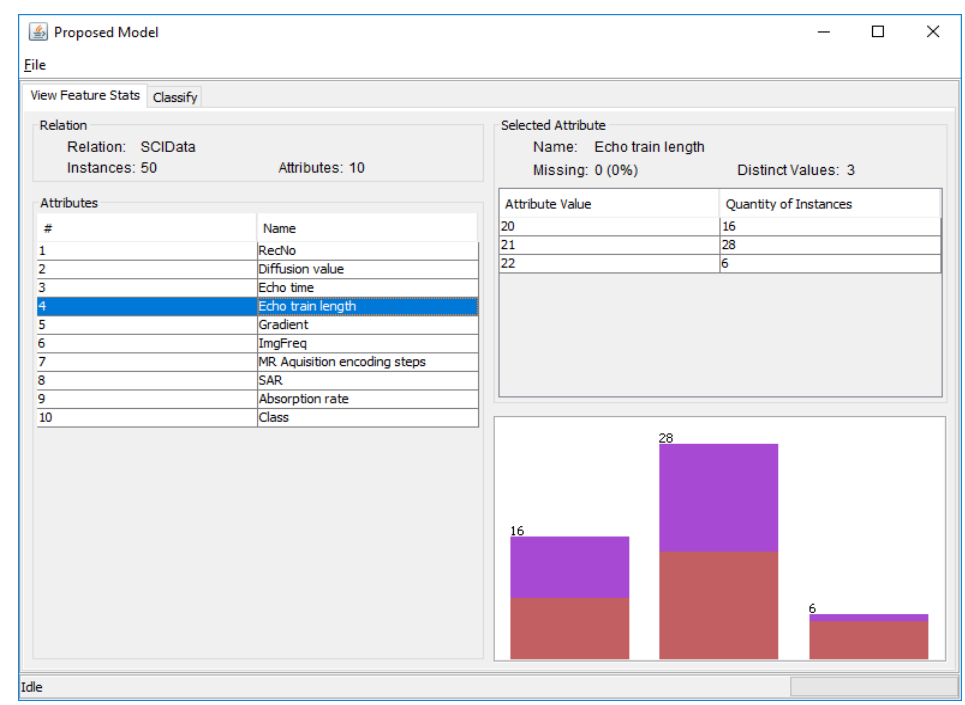

Figure 8. Spinal cord Injury data segments and its statistical properties. 
Figure 9, describes the multi-level segmentation dataset of the training spinal cord image data. In the figure, segmentation training data and its statistical properties of each attribute such as msising value, distinct values etc are given to validate the spinal cord regions. Table 1, gives the information of the proposed method comparison to the existing method. In the table, proposed method has high computational improvement compared to traditional method.

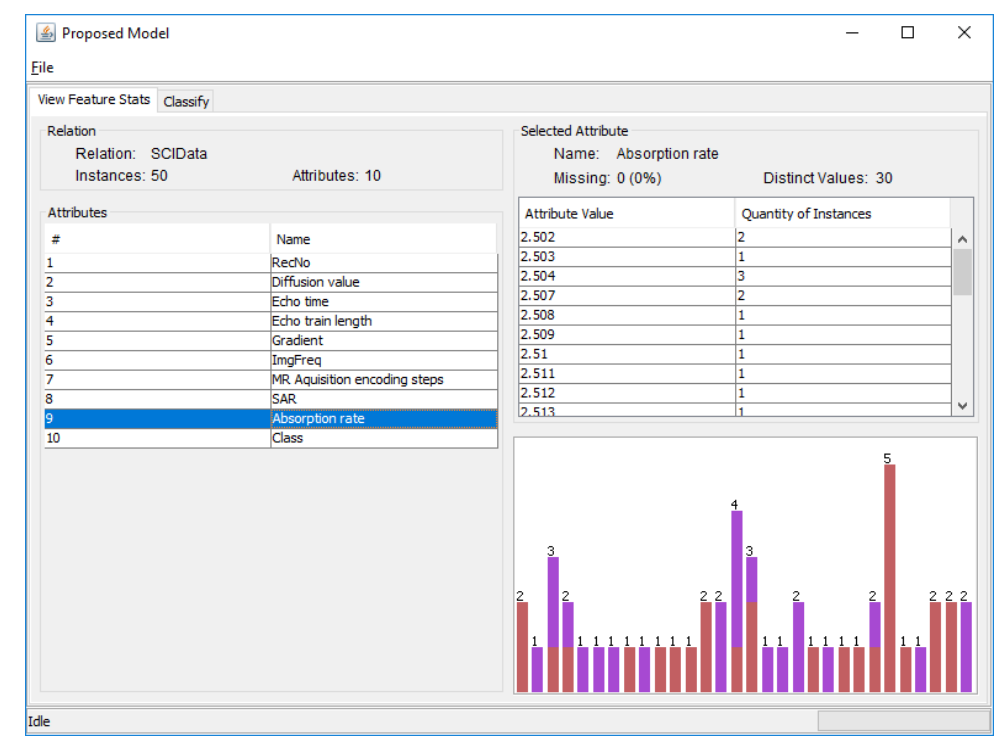

Figure 9. SCI Training features and its sparsity values

\begin{tabular}{cccc}
\hline SNO & Parameter & Method & \% Improvement \\
\hline 1 & SCI & SVM & 70 \\
2 & SCI-MLS & CT-MRI & 90 \\
\hline
\end{tabular}

Table 1. Comparison of proposed method to traditional methods.

\section{CONCLUSION}

Finally, here we achieve good results and increasing the efficiency $80 \%$ and accuracy is to increase 70\%. Using this AUTOMATIC SCI-MLS method is the best method to find injuries on the spinal card.

\section{FUTURE SCOPE}

Estimation of the CSA, which has been shown to be a marker of decay, and examination inside locales of intrigue (GM, WM tracts) are of specific significance. By encouraging longitudinal and multifocus ponders, these strategies ought to significantly help in additionally understanding the spinal line way physiology, and to grow new HYBRID SEGMENTATION criteria for later location of subclinical development, for anticipation forecast or potentially tolerant administration.

\section{REFERENCES}

[1] S. K. Ahammad and V. Rajesh, "Image Processing based Segmentation Techniques for Spinal Cord in MRI," Indian Journal of Public Health Research \& Development, vol/issue: 9(6), 2018.

[2] N. Archip, et al., "A knowledge-based approach to automatic detection of the spinal cord in CT images," IEEE Transactions on Medical Imaging, vol/issue: 21(12), pp. 1504-1516, 2002.

[3] Z. Peng, et al., "Automated vertebra detection and segmentation from the whole spine MR images," in Engineering in Medicine and Biology Society, 2005.IEEE-EMBS 2005. 27th Annual International Conference of the, pp. 25272530, 2006.

[4] Stroman P. W., et al., "The current stateof-the-art of spinal cord imaging: methods," Neuroimage, vol. 84, pp. 1070-1081, 2014

[5] L. Fradet, et al., "Morphometrics of the entire human spinal cord and spinal canal measured from in vivo highresolution anatomical magnetic resonance imaging," Spine, vol/issue: 39(4), pp. E262-E269, 2014. 
[6] V. Rajesh, et al., "A Telemedicine Technology for Cardiovascular Patients Diagnosis Feature Using KNN-MPM Algorithm," vol. 46, pp. 72-77.

[7] S. H. Ahammad, et al., "Identification of Cervical Spondylosis disease on Spinal Cord MRI Image using Convolutional Neural Network-Long Short-Term Memory (CNN-LSTM) Technique," vol. 46, pp. 108-124.

[8] K. Saikumar, et al., "A Deep Learning Process for Spine and Heart Segmentation using Pixel-Based Convolutional Networks," Journal of International Pharmaceutical Research, vol. 46, pp. 278-282, 2019.

[9] H. Kearney, et al., "Improved MRI quantification of spinal cord atrophy in multiple sclerosis," Journal of magnetic resonance imaging, vol/issue: 39(3), pp. 617-623, 2014.

[10] R. C. Gonzalez and R. E. Woods, "Digital Image Processing," 2nd ed, Beijing, Publishing House of Electronics Industry.

[11] S. Sridhar, "Digital Image Processing," New Delhi: Oxford University Press, 2013.

[12] W. X. Kang, et al., "The Comparative Research on Image Segmentation Algorithms," IEEE Conference on ETCS, pp. 703-707, 2009. 